University of Nebraska - Lincoln

DigitalCommons@University of Nebraska - Lincoln

Robert G. Fuller Publications and Presentations Research Papers in Physics and Astronomy

March 1997

\title{
The Ubiquitous Coffee Filter
}

Norman F. Derby

Bennington College, Bennington, VT

Robert Fuller

rfuller@neb.rr.com

Phil W. Gronseth

Colorado Springs Christian High School, Colorado Springs, CO

Follow this and additional works at: https://digitalcommons.unl.edu/physicsfuller

Part of the Physics Commons

Derby, Norman F.; Fuller, Robert; and Gronseth, Phil W., "The Ubiquitous Coffee Filter" (1997). Robert G. Fuller Publications and Presentations. 40.

https://digitalcommons.unl.edu/physicsfuller/40

This Article is brought to you for free and open access by the Research Papers in Physics and Astronomy at DigitalCommons@University of Nebraska - Lincoln. It has been accepted for inclusion in Robert G. Fuller Publications and Presentations by an authorized administrator of DigitalCommons@University of Nebraska - Lincoln. 


\title{
The Ubiquitous Coffee Filter
}

\author{
Norman F. Derby \\ Bennington College, Bennington, VT 05201; derbynf@bennington.edu
}

Robert G. Fuller

University of Nebraska, Lincoln, NE 68588-0111; rfuller@unlinfo.unl.edu

\author{
Phil W. Gronseth \\ Colorado Springs Christian High School, Colorado Springs, CO 80918; Gronseth@kktv.com
}

W hat analyst would have predicted that $\mathrm{Mr}$. Coffee $\mathrm{C}^{\mathrm{TM}}$ would diversify into the scientific supplies market? Nevertheless, that coffeemaker's filters, with their flat bottoms and fluted sides, have been popular in physics classroom experiments for some time now. Besides being cheap and readily available, coffee filters allow students to work experimentally with non-constant forces and, because the filters can be easily stacked without changing their aerodynamic profile, the variation in drag force with velocity can be studied. Since stacks of a few filters fall at speeds of about a meter per second and even a stack of ten reaches terminal velocity rapidly, they can be observed with no more equipment than a stopwatch and meterstick. However, for those who prefer more high-tech approaches, the filters can be dropped directly onto sonic rangers and the data imported to a computer, or the filters can be videotaped and their motion digitized and analyzed using software developed for just such purposes. ${ }^{1}$

\section{Theory}

For reference, we present some equations describing falling coffee filters. From the Reynolds number, there is reason to expect that the drag force acting on the filters during their fall is proportional to the square of their velocity, ${ }^{2}$
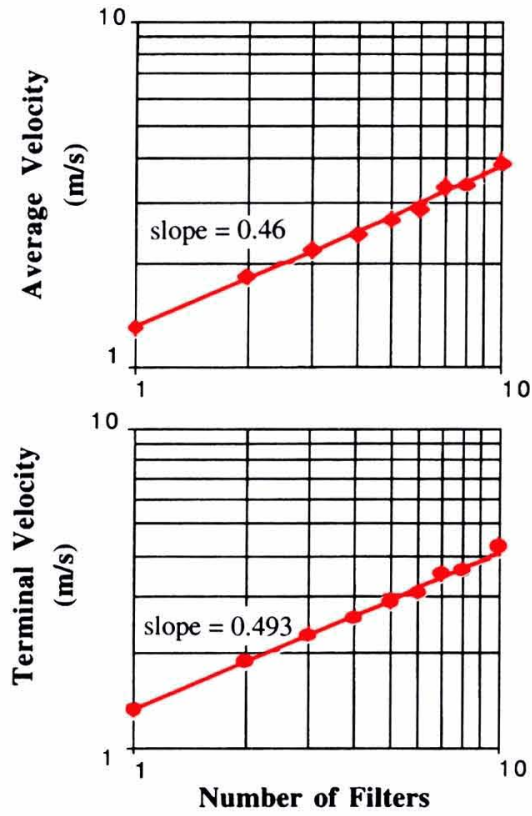

Fig. 1. (top) Average velocities of coffeefilter stacks dropped $15 \mathrm{~m}$ and best-fit line. (bottom) Terminal velocity of filters, as derived from the average velocities, and best-fit line.

$$
F_{\text {drag }}=K v^{2}
$$

When a falling object reaches terminal velocity, $v_{T}$, the net force on it is zero, so

$$
F_{\text {drag }}=m g \quad \text { or } \quad K=m g / v_{T}^{2}
$$

Thus, the equation of motion of a falling filter is

$$
\frac{d v}{d t}=g\left[-1+\left(\frac{v}{v_{T}}\right)^{2}\right]
$$

Equation (3) is an interesting one for beginning students, both because it is a separable differential equation that can be integrated directly, and because the integration gives students a chance to apply their knowledge of hyperbolic trig functions for perhaps the first time. The solution for velocity as a function of time is

$$
v=-v_{T} \tanh \left(\frac{g t}{v_{T}}\right)
$$

This can be integrated once more to find position as a function of time

$$
y=y_{0}-\left(\frac{v_{T}^{2}}{g}\right) \ln \left[\cosh \left(\frac{g t}{v_{T}}\right)\right]
$$

See Appendix for a discussion of these hyperbolic functions.

\section{Experiment: Long Distances}

The Guinness book ${ }^{3}$ does not mention a distance record for dropping coffee filters, but the following experiment may have set one. Stacks of filters were dropped down the well of a circular stairwell in a university library, a distance of $15 \mathrm{~m}$. A single experimenter can drop a stack of filters 


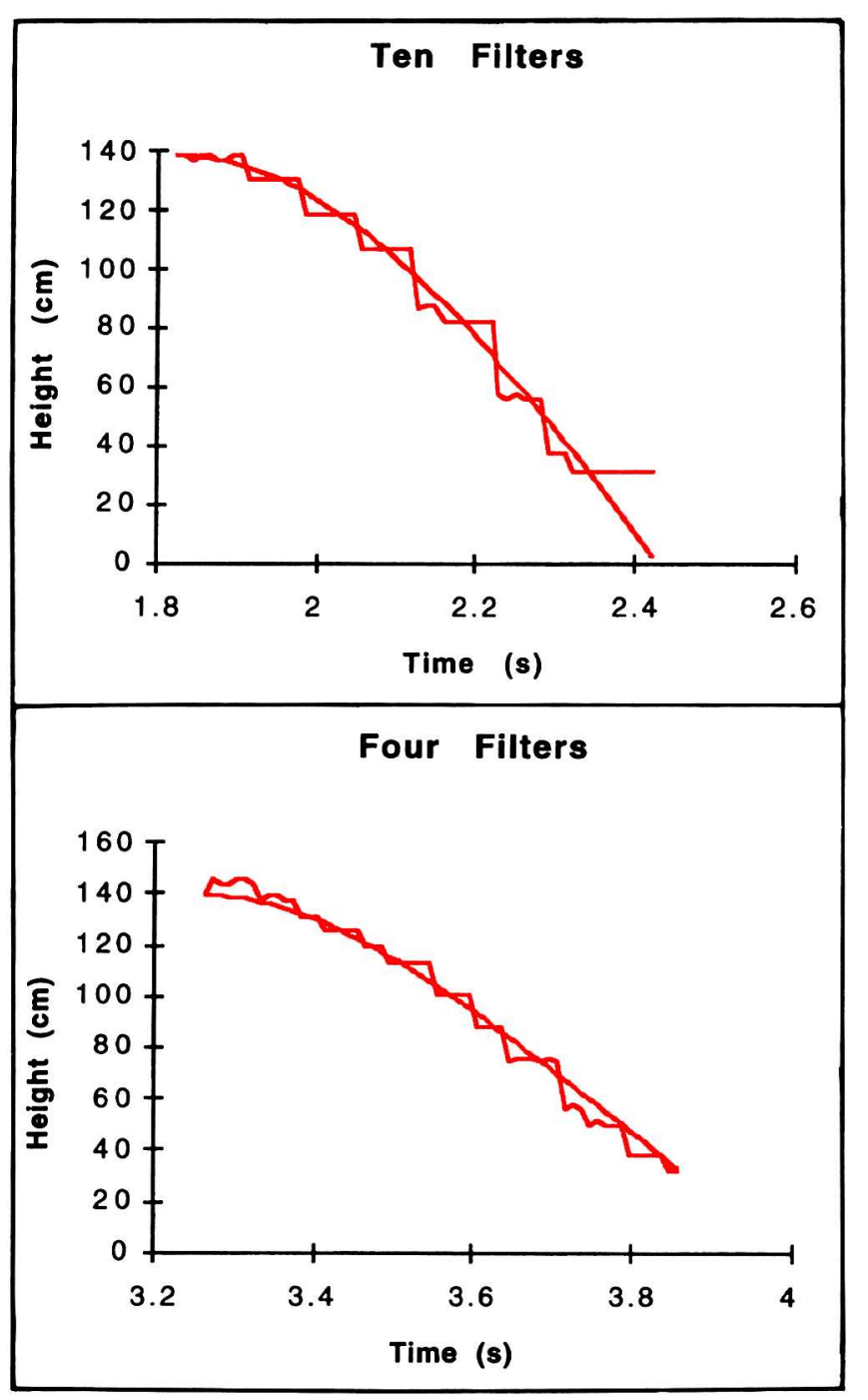

Fig. 2. Sonic-ranger measurements of height of falling stacks of coffee filters. Smooth curves were calculated from Eq. (5), using terminal velocities obtained from Fig. 1.

and time their fall with a stopwatch to better than $1 \%$ accuracy. The aerodynamic stability of coffee filters is noteworthy - even single filters follow a remarkably straight line of descent, generally landing within a $1-\mathrm{m}$ circle despite air currents. Although the quantity measured for each stack is the average velocity of its fall, small stacks achieve terminal velocity so quickly that the distinction between average and terminal velocity can be ignored in a rough analysis. At terminal velocity, the net force acting upon the filters is zero, $m g=$ $K v_{T}^{2}$, so a $\log -\log$ plot of terminal velocity versus the number of the filters should be a straight line with a slope of 0.5. Such a graph is shown in Fig. 1 (top). The least-squares fit line has a slope of $0.46-$ not bad considering the simplicity of the experiment and the analysis.

Of course heavier stacks take significant time to reach terminal velocity, so a more careful self-consistency analysis could be done as follows: 1) assume that the drag force is proportional to the square of the velocity; 2) use that assumption to calculate the terminal velocity from the average velocity; 3) make a log-log plot of the calculated terminal velocities against mass to verify that the slope is 0.5 and thus confirm the assumption.

To perform this self-consistency analysis, we define $T \equiv$ time for fall; then from Eq. (5)

$$
\bar{v}=\frac{\Delta y}{T}=-\left(\frac{v_{T}^{2}}{g T}\right) \ln \left[\cosh \left(\frac{g T}{v_{T}}\right)\right]
$$

If we then define a parameter $\alpha \equiv$ $g T / v_{T}$, this last equation can be written as

$$
\left(\frac{-\bar{v}}{g T}\right)=\frac{\ln (\cosh \alpha)}{\alpha^{2}}
$$

From the experimental data we can compute the left-hand side of Eq. (7), and can then solve (7) numerically for $\alpha$, thus determining $v_{T}$. A graph of $v_{T}$ determined in this way versus the number of filters is shown in Fig. 1 (bottom). The least-squares fit line has a slope of 0.493 with a standard error of 0.012 .
For those who find this last method too indirect, the experiment can be performed with a second experimenter at the bottom of the stairwell measuring the time for the last one or two meters of fall. The spread in landing positions makes use of standard photogates or sonic rangers impractical, but such difficulties can be overcome with a little creativity.

\section{Experiment: Short Distances}

To examine the behavior of the filters before they reached terminal velocity, we tried dropping them directly onto a sonic ranger from a height of $2 \mathrm{~m}$, which was about the maximum usable range for these sonic rangers. The experimental procedure was first to start collecting data from the sonic ranger and then to drop the filters. A sample of raw data is shown in Fig. 2. The theoretical curves shown are based on Eq. (5), with the values for $v_{T}$ being those we obtained from the long-distance drops. The theoretical curves were adjusted left or right, depending on the starting time for the drop, but otherwise there were no adjustable parameters. The fit looks good, reassuring us that Eq. (1) is correct in this situation.

\section{Assignments and Activities}

With coffee-filter experiments, it is possible to engage students in a variety of activities ranging from simple to sophisticated and with different degrees of structure. Some of our most interesting experiences involved giving assignments with minimal structure to see what our students could do. In coffeefilter dropping, the time of fall depends both on the distance and the number of filters dropped, two variables easily manipulated by students, so these experiments provide a good way to challenge students to separate and control variables.

Consider the following in-class activity given to college freshmen in the first semester of a calculus-based physics course; time allotted was about 50 minutes.

Your team is given four coffee filters. Pan balances, metersticks, and sonic rangers are available. Your task is to conduct some experiments and figure out what 
Table I. Data for Coffee-Filter Homework Assignment.

\begin{tabular}{ccc}
\hline Number of Filters & Distance (m) & Time (s) \\
\hline 1 & 0.25 & 0.46 \\
2 & 0.50 & 0.60 \\
3 & 0.75 & 0.70 \\
4 & 1.00 & 0.80 \\
1 & 2.00 & 3.15 \\
4 & 1.4 & 1.1 \\
2 & 2.00 & 2.22 \\
3 & 0.20 & 0.22 \\
4 & 0.50 & 0.42 \\
3 & 2.00 & 1.80 \\
1 & 1.25 & 2.00 \\
2 & 1.00 & 1.13 \\
1 & 0.6 & 1.00 \\
2 & 0.25 & 0.32 \\
4 & 0.20 & 0.18 \\
3 & 1.5 & 1.37 \\
\hline
\end{tabular}

forces act on the filters as they fall through the air. You have until the end of the period to carry out your experiments, report your data, and summarize your results.

Twelve teams of three to four students were given this activity. They had difficulty in figuring out how to get a measurement of any kind. Their reports were virtually useless. However, since the students now had some experience dropping coffee filters, we decided to probe further by giving them a followup homework assignment, which was intended to help them understand their previous in-class work without being too directive.

A group of university freshmen decided to figure out exactly how coffee filters fall through air near sea level. They dropped coffee filters from rest, individually and with some stuck inside one another, and let them fall various distances. They measured the time of fall. Their data, as they collected it, are given in Table I. Prepare a written report to explain the data and comment on the experimental procedures. What forces seem to be acting on these coffee filters?

The position-time data in Table I simply describe four straight lines with different slopes due to the different masses, but the order of the points was deliberately scrambled. Students in our course had all completed at least a semester of calculus in which they used Mathe- matica $^{\mathrm{TM}}$ and all of them had personal computers with Mathematica $^{\mathrm{TM}}$ and a spreadsheet program. They had worked with spreadsheets on a number of previous assignments. It might seem obvious that the data in this table should be sorted and/or graphed in order to analyze it conveniently. It might also seem obvious that a spreadsheet would make this easy to do. Not so. For many of the students, this homework assignment was both mysterious and impossible.

In a sample of 12 answers to this question, only four students attempted any kind of graph and only one of those was properly done-and that one was done without use of a spreadsheet. Students observed that the data were mixed up, but that was generally where they stopped. Some attempted verbal explanations that were very self-revealing, using technical terms in a meaningless way; for example "The coffee filters are

\begin{tabular}{|ccc|}
\hline \# of coffee filters & distance of drop $(\mathbf{m})$ & time of fall (s) \\
\hline 4 & 1 & 0.8 \\
4 & 1.4 & 1.1 \\
4 & 0.5 & 0.42 \\
4 & 0.2 & 0.18 \\
\hline
\end{tabular}

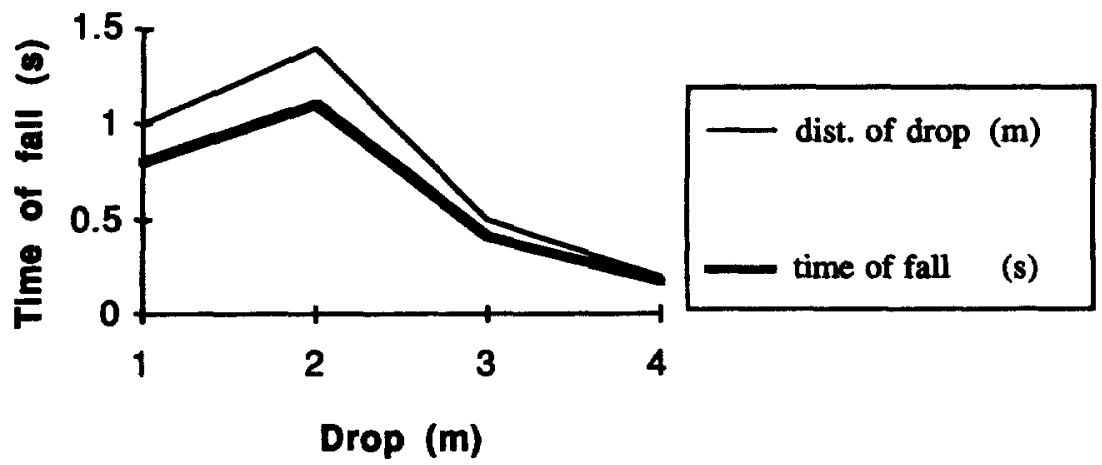

Fig. 3. Student graph purporting to show "time of fall of a coffee-filter stack versus distance of the drop." Graph was obtained from a spreadsheet program using data shown. falling at a constant acceleration, the force of gravity.... The reason the filters start falling faster is because [of] the greater mass. If the acceleration is constant, the mass is the only thing that affects the force on an object." An example of one of the spreadsheet graphs purporting to show "time versus distance of the drop for a stack of four filters" is shown in Fig. 3. Apparently because the context of the question was unfamiliar, the student was unable to recognize that the spreadsheet graphs were utterly meaningless.

We hasten to point out that students in our calculus-based physics course were a select group of college freshmen. Many of them had high SAT math scores and all were selected from a group of volunteers who in nearly all cases had already completed highschool physics, so their answers cannot be attributed to poor preparation or incompetence. In a standard course, they would be mostly A students.

\section{Observations}

Coffee filters are inexpensive items that can be used in a variety of ways in the physics classroom. Because of the ease with which experiments can be performed, they lend themselves to unstructured activities in which students must discover for themselves the principles of organization that are needed to 
collect useful data. Our experience, however, is a warning, if one is needed, that understanding how to follow lab instructions and/or answer standard homework questions correctly is not necessarily the same thing as real understanding - which is what we are all aiming for.

\section{References}

1. Daniel J. Bodony and Dan MacIsaac, AAPT Announcer 25, 72 (December 1995).

2. Ascher H. Shapiro, Shape and Flow (Anchor Books, New York, 1961).

3. Norris McWhirter, The Guinness Book of World Records 1997 (Bantam Books, New York, 1997).

\section{Appendix}

Note the physical significance of these hyperbolic functions. In Eq. (4) for short times, $\frac{g t}{v_{T}}<1$. For this range, $\tanh \left(\frac{g t}{v_{T}}\right) \approx \frac{g t}{v_{T}}$. In this approximation, Eq. (4) becomes $v=-g t$, which is what we would expect from free fall before air resistance is important.

In Eq. (5) there is a hyperbolic cosine, which can be expanded as:

$$
\cosh \alpha=1+\frac{\alpha^{2}}{2 !}+\frac{\alpha^{4}}{4 !}+\ldots
$$

Therefore, for small time:

$$
y \approx y_{0}-\left(\frac{v_{T}^{2}}{g}\right) \ln \left[1+1 / 2\left(\frac{g t}{v_{T}}\right)^{2}\right]
$$

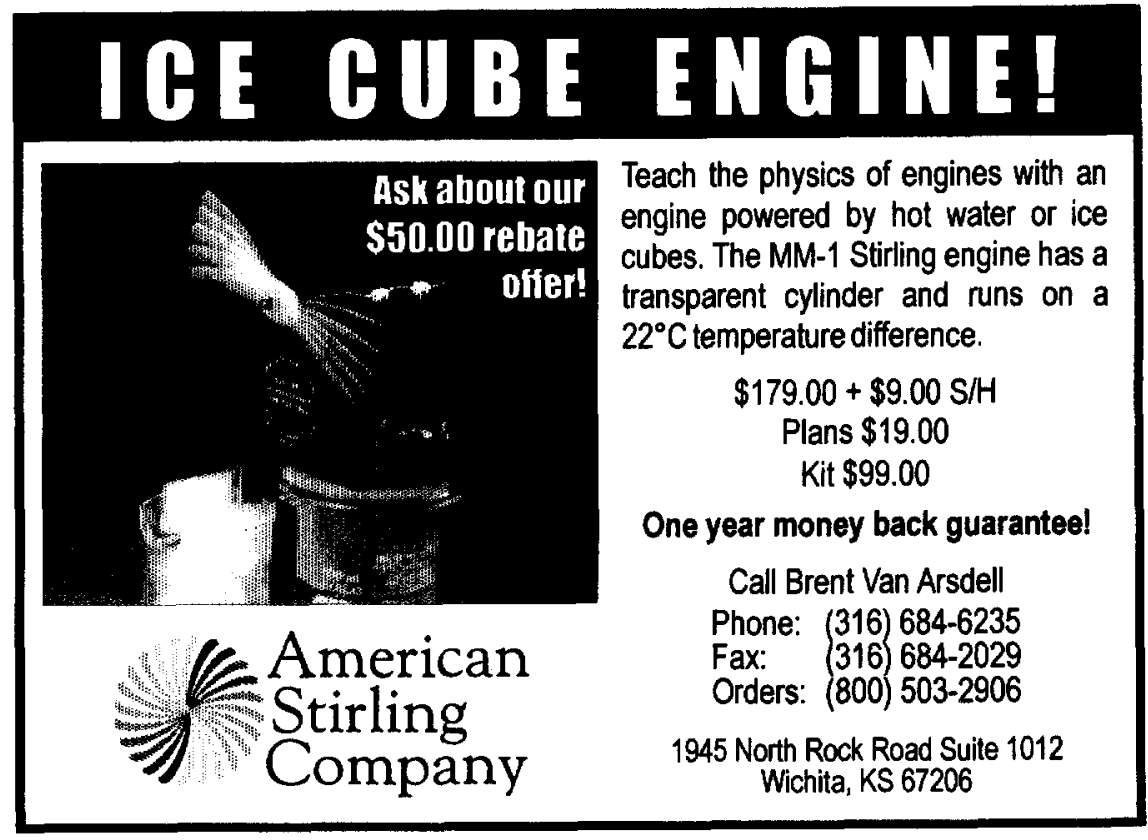

Since $\ln (1+x) \approx x$ for small $x$ :

For large $t$,

$$
y \approx y_{0}-\left(\frac{v_{T}^{2}}{g}\right) 1 / 2\left(\frac{g t}{v_{T}}\right)^{2}=y_{0}-1 / 2 g t^{2}
$$

This is the equation for $y(t)$ for an object in free fall at an early time before air resistance is important.

To investigate Eq. (5) for large $t$, use the exponential definition of cosh:

$$
\begin{aligned}
y & =y_{0}-\left(\frac{v_{T}^{2}}{g}\right) \ln \left(\frac{e^{\alpha}+e^{-\alpha}}{2}\right) \\
\text { where } \alpha & =\left(\frac{g t}{v_{T}}\right) .
\end{aligned}
$$

$$
\begin{array}{r}
y \rightarrow y_{0}-\left(\frac{v_{T}^{2}}{g}\right) \ln 1 / 2 e^{\alpha}= \\
y_{0}-\left(\frac{v_{T}^{2}}{g}\right)\left(\ln 1 / 2+\frac{g t}{v_{T}}\right)= \\
y_{0}-v_{T} t+0.69\left(\frac{v_{T}^{2}}{g}\right)
\end{array}
$$

For large $t$, the object's position is dominated by the distance it would have fallen at constant terminal speed, but is less than this amount by a constant, which, in effect, is the intercept of the $y(t)$ curve.

\section{Does Your Baby Know Things Your Students Don't?}

Professor Fritz Siemsen (Johann Wolfgang Goethe-Universität, Frankfurt am Main, Germany) sent us this picture of his baby daughter reaching for her image in the mirror. Note, however, that the baby apparently sees the image behind the mirror.

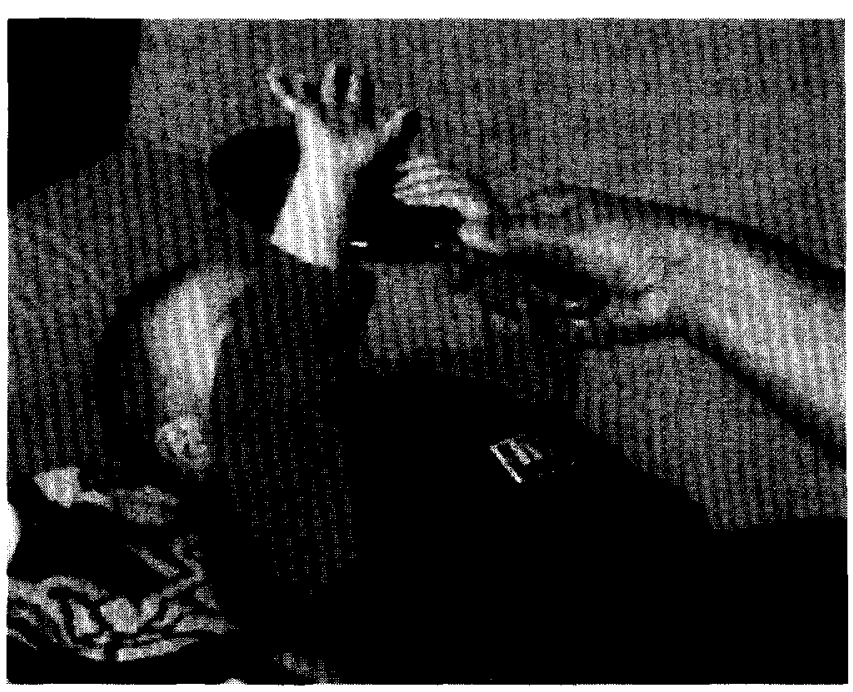

